\title{
The young, wide and very low mass visual binary Lambda Orionis $167^{\star}$
}

\author{
D. Barrado y Navascués ${ }^{1}$, A. Bayo ${ }^{1}$, M. Morales-Calderón ${ }^{1}$, N. Huélamo ${ }^{1}$, J. R. Stauffer ${ }^{2}$, and H. Bouy ${ }^{3}$ \\ ${ }^{1}$ Laboratorio de Astrofisica Espacial y Fisica Fundamental, LAEFF-INTA, Madrid, Spain \\ e-mail: barrado@laeff.inta.es \\ 2 IPAC, Caltech, Pasadena, USA \\ e-mail: stauffer@ipac.caltech.edu \\ 3 Astronomy Department, University of California, Berkeley, USA \\ e-mail: hbouy@astro.berkeley.edu
}

Received 8 February 2007 / Accepted 11 April 2007

\section{ABSTRACT}

\begin{abstract}
Aims. We look for wide, faint companions around members of the $5 \mathrm{Myr}$ Lambda Orionis open cluster. Methods. We used optical, near-infrared, and Spitzer/IRAC photometry.

Results. We report the discovery of a very wide very low mass visual binary, LOri167, formed by a brown dwarf and a planetarymass candidate located at 5 arcsec, which seems to belong to the cluster. We derive $T_{\text {eff }}$ of 2125 and $1750 \mathrm{~K}$. If they are members, comparisons with theoretical models indicate masses of $17_{-2}^{+3}$ and $8_{-1}^{+5} M_{\text {jup }}$, with a projected separation of $2000 \mathrm{AU}$.

Conclusions. Such a binary system would be difficult to explain in most models, particularly those where substellar objects form in the disks surrounding higher mass stars.
\end{abstract}

Key words. open clusters and associations: individual: Collinder 69 - stars: low-mass, brown dwarfs - stars: binaries: visual stars: pre-main sequence

\section{Introduction}

During the past few months, several very low mass binaries have been reported in the literature (Close et al. 2003; Chauvin et al. 2004; Kraus et al. 2005, 2006). They are relatively close visual binaries, with angular separations of a fraction of an arcsecond and projected distances of a few tens of AU or less. On the other hand, other authors (Billères et al. 2005; Luhman et al. 2004, 2005; Jayawardhana \& Ivanov 2006; Close et al. 2006; Bouy et al. 2006; Caballero et al. 2006; Caballero 2007) have found a population of very low mass binaries in several young associations or in the field, with separations in the range 100-250 AU. These binaries might include either one or two brown dwarfs, or might also include a planetary-mass object, whose mass is predicted by theoretical models to be below the deuterium-burning limit at about $13 M_{\text {jup }}$ masses. Such binaries are extremely important because they challenge the latest formation scenarios for very low mass objects and might indicate that very low mass brown dwarfs and planetary-mass objects form by collapse and fragmentation of molecular clouds, just as stars do, and that the minimum mass for the process is much lower than what had been previously thought.

We have discovered a close $\left(5^{\prime \prime}\right)$ optical binary in the Lambda Orionis cluster (Collinder 69). This association is about 5 Myr (Dolan \& Mathieu 1999, 2001; Barrado y Navascués et al. 2007) and is located at about $400 \mathrm{pc}$. If the pair is physically associated both with each other and with the cluster, they would

$\star$ Based on observations carried out with the CFHT, the WHT, the CAHA, the Spitzer and the Keck telescopes. be one of the lowest mass binary systems and would have one of the widest separations reported in the literature, about 2000 AU.

\section{Analysis}

We combined optical, near infrared, and Spitzer/IRAC photometry in order to look for faint, very low mass members of the Collinder 69 or Lambda Orionis open cluster (Dolan \& Mathieu 1999, 2001). We first combined optical data in the Ic filter taken at the CFHT in October 1999 with the $12 \mathrm{~K}$ camera (Barrado y Navascués et al. 2004) and with deep $J$ imaging taken at the WHT in February 2003 with the INGRID instrument (Barrado y Navascués et al. 2005b). The use of a color-color diagram allowed us to select a sample of very faint, red objects. These results will be reported elsewhere. In particular, one of them was very close to the brown dwarf candidate LOri167 (Fig. 1, see also Fig. 1 in Barrado y Navascués et al. 2005b), whose mass, if it is a member, is just above the planetary-mass borderline at $13 M_{\text {jup }}$, in the range $15-20 M_{\text {jup }}$ - Dusty $5 \mathrm{Myr}$ isochrone by Chabrier et al. (2000), which is appropriate for its effective temperature (see below). Subsequent follow-up with the IRAC camera onboard the Spitzer infrared telescope has helped us to confirm that this object falls in the cluster sequence (see Fig. 2).

We also observed this area in the filters $J, H$, and $K$ s in previous campaigns with WHT/INGRID (November 2002) and the Calar Alto $3.5 \mathrm{~m}$ telescope and Omega 2000 (October 2005). All the photometric data were analyzed in the same way, 
Table 1. Data for the LOr167 visual binary.

\begin{tabular}{lllllllll}
\hline \hline LOri & alpha (2000.0) delta & $R^{(1)}$ & $I^{(1)}$ & $J^{(2,3)}$ & $H^{(2)}$ & $K \mathrm{~s}^{(2)}$ & {$[3.6]$} & {$[4.5]$} \\
\hline 167 & 83.80913549 .9020901 & 23.860 .64 & 20.900 .02 & 17.880 .03 & 17.150 .03 & 16.620 .03 & 16.040 .03 & 16.370 .09 \\
167 comp & 83.81047829 .9020519 & - & 23.230 .18 & 20.190 .19 & 19.410 .17 & 18.310 .14 & 16.850 .06 & 16.760 .14 \\
\hline
\end{tabular}

(1) RI photometry from CFHT September 1999 (Barrado y Navascués et al. 2004).

(2) $J H K$ s data from WHT/INGRID November 2002.

(3) $J=17.78 \pm 0.02$ and $20.36 \pm 0.08$ for the LOri167 and LOri167comp in the deep $J$ image (WHT/INGRID February 2003).

LOri167 $J=18.01 \pm 0.03, H=17.17 \pm 0.07, K \mathrm{~s}=16.83 \pm 0.09,[3.6]=15.935 \pm 0.063,[4.5]=16.060 \pm 0.129$, in Barrado y Navascués et al. (2007). The near IR data were taken with Omega2000 at Calar Alto Observatory (October 2005).

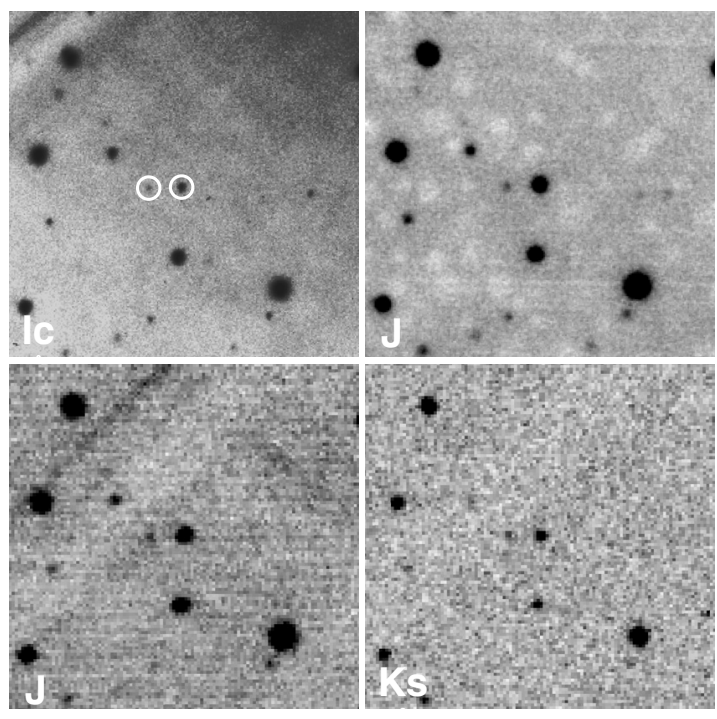

Fig. 1. $45^{\prime \prime} \times 45^{\prime \prime}$ images around LOri167, corresponding to the CFHT in october 1999 and the WHT in february 2003.

using aperture photometry within the $\operatorname{IRAF}^{1}$ environment. For the IRAC data, we used an aperture of 2 pixels, to reduce the errors of the faint companion. For the near-IR data, the calibration was achieved using either standard stars or the 2MASS catalog and the stars present in the field of view. The data are listed in Table 1.

\subsection{Membership in the cluster}

Using all available data (see Table 1), we were able to build the spectral energy distribution (SED) of both objects (Fig. 3). Clearly, they are cool objects, with the visual companion much cooler than LOri167. We tried to derive proper motions in our images, but the accuracy of the astrometry goes with the inverse of the signal-to-noise ratio, and the data do not go deep enough for these two objects.

Despite the faintness of LOri167, we tried to secure a lowresolution spectrum on a couple of occasions, both with the Keck telescope and the NIRSPEC spectrograph, in order to derive a spectral classification (November 2004 and December 2005). Unfortunately on both occasiones the weather did not cooperate (bad seeing, clouds), and we were only able to obtain a noisy, low-quality spectrum. Comparison with spectral templates suggests an M9-L2 spectral type for LOri167. If it is a member of Collinder 69 (very likely), LOri167 would have, from

${ }^{1}$ IRAF is distributed by National Optical Astronomy Observatories, which is operated by the Association of Universities for Research in Astronomy, Inc., under contract to the National Science Foundation, USA.

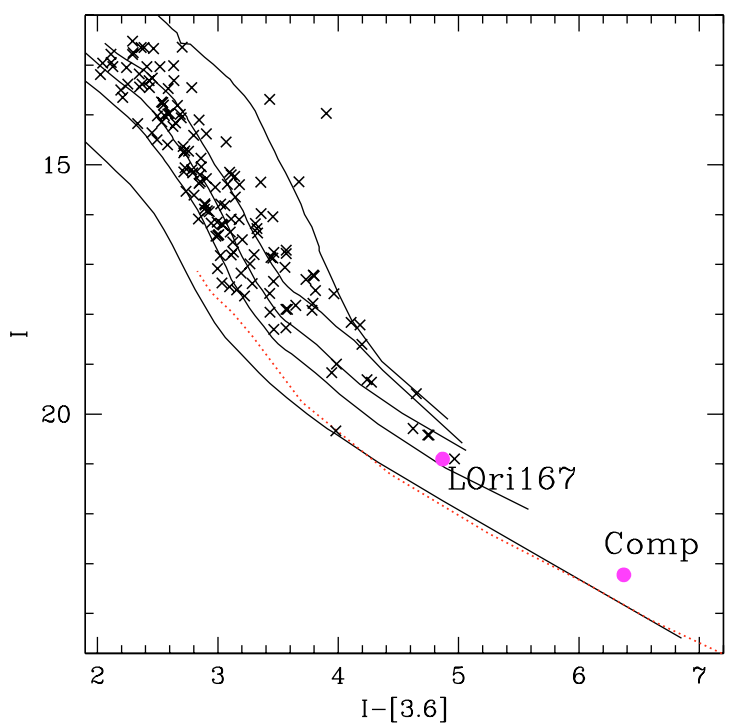

Fig. 2. Color-magnitude diagram for the 5 Myr cluster Collinder 69. The LOri167 brown dwarf and its visual companion appear as thick, big circles. NextGen isochrones of 1, 5, 10, 20 and $100 \mathrm{Myr}$ by Baraffe et al. (1998) appear as solid lines ( $L$ data instead [3.6]). We have also included a Dusty $5 \mathrm{Myr}$ isochrone - dotted line.

comparison with theoretical isochrones, a mass in the range 15$20 M_{\text {jup }}$, as stated before. These estimates also include a maximum age of 7-8 Myr, corresponding to a plausible estimate of the oldest stars in the cluster (see Barrado y Navascués et al. 2005a, and references therein). We fitted both black bodies and theoretical atmospheric models (Allard et al. 2001, 2003) to the SEDs and derived $T_{\text {eff }}=2125 \mathrm{~K}$, in full agreement with the values predicted by the photometry, the isochrones, and the spectral type. Thus, we are confident of the membership of this object and its substellar nature.

The visual companion (LOri167comp) is probably too faint for useful low-resolution IR spectroscopy $(J=20.19)$. If a member, it would probably be an object with mid to late $\mathrm{L}$ spectral type. An SED fitting indicates $T_{\text {eff }}=1750 \mathrm{~K}$, corresponding to L6 on the temperature scale of Basri et al. (2000). This temperature and the position in the color-magnitude diagrams fully agree with membership. The predicted mass, using a $5 \mathrm{Myr}$ Dusty isochrone by the Lyon group, is in the range 7-13 $M_{\text {jup }}$, with an optimal value, as derived from $T_{\text {eff }}$, of $8 M_{\text {jup }}$. A $10 \mathrm{Myr}$ isochrone (much older than the age of the cluster) would produce masses in the range $11-15 M_{\text {jup }}$. The high values in mass come from the Spitzer/IRAC magnitudes and the Dusty isochrone might indicate the possible presence of a disk or ring, although the magnitudes in the theoretical models are far from perfect, especially in the almost unexplored domain of the extremely faint, red, and low mass objects. 

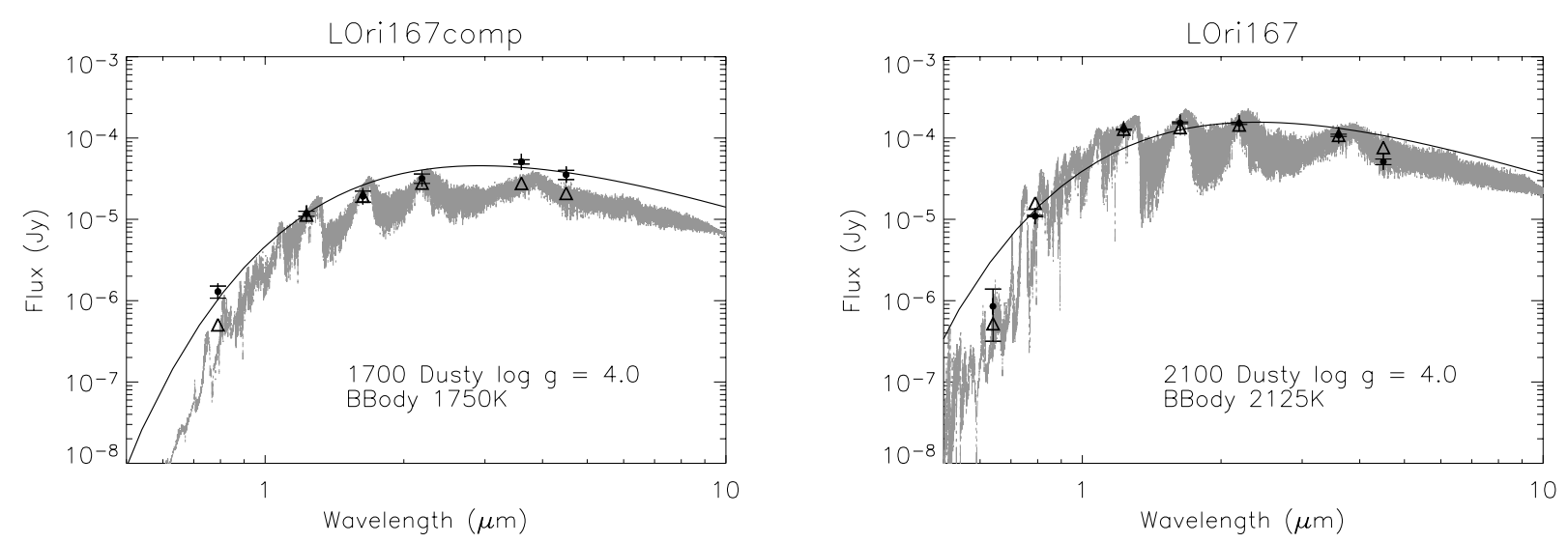

Fig. 3. Spectral energy distributions for our targets, using optical, near-IR and Spitzer/IRAC data. Solid circles represent the actual measurements, and open triangles correspond to the synthetic photometry from the models.

\subsection{Are the two objects a physical pair?}

We estimated the contamination by unrelated visual companions by deriving the probability of finding an object with the luminosity and colors of LOri167comp. The total number of objects brighter by half a magnitude in both $I$ and $J$, and with $I-J>$ 2.8 , is equal to three. This estimate is very conservative since the maximum uncertainties in each band are below 0.16 mag in $I$ and 0.07 mag in $J$. Counting only the objects with $I$ and $J$ magnitudes and $(I-J)$ colors within these uncertainties, we find only one object, which is LOri167comp itself. Since our total field of view is $4.1^{\prime} \times 4.1^{\prime}$, the probability of finding such a faint and red object in a 5 arcsec circle around any object is three times $1.3 \times$ $10^{-3}$, about $0.4 \%$. In this field of view, there are 11 probable members of the Collinder 69 cluster (Barrado y Navascués et al. 2005b, 2007), but only three are in the substellar domain (such as LOri167), with magnitudes less than $I c=17.55$. Therefore, the total probability of finding a visual binary by chance mimicking this type of binary (two brown dwarfs or a brown dwarf plus a planetary-mass object) is slightly higher than $1 \%$. Because this probability is reasonably low, we believe it favors the planetarymass object being a real companion. Such a calculation is only tentative and must be considered with caution. We are well aware of the dangers of applying a posteriori statistical tests, so are simply arguing that the data are consistent with the planetarymass candidate being a companion, although it will be difficult to prove (or disprove) this association with existing instrumentation. And we emphasize that LOri167comp is well below the completeness limit of our optical and infrared surveys.

Therefore, this probabilistic argument based on photometric analysis of the objects in the field supports the hypothesis that it is, indeed, a physical pair formed by a low mass brown dwarf and a planetary-mass object. A population of low-mas binaries with a 100-250 AU separation has been discovered in UpSco and other young associations (Bouy et al. 2006, and references therein) or in the field (Billères et al. 2005). Moreover, Caballero et al. (2006) have found that the visual binary SE70+SOri68 might be real, with a separation of $4.7^{\prime \prime}$, equivalent to $1700 \mathrm{AU}$. Due to the separation and low mass, even if they are bound, most of these wide binaries will be disrupted soon, either by the interactions with other cluster members or by field stars and the host association moving in the galactic gravitational field. However, Caballero (2007) has recently discovered two low mass stars with common proper motion and a projected separation of 1800 AU. During the revision of this paper, the widest low mass binary was announced, with a projected separation of about $5100 \mathrm{AU}$ and a mass for the primary lower than $0.1 M_{\odot}$ (Artigau et al. 2007). Since this seems to be an old, field system with stellar masses, it suggests that at least a few wide binaries may survive for very long periods of time. Additional results have also been published by Kraus \& Hillenbrand (2007) in three young associations. In any case, LOri167 would have a binding energy about 20 times lower than Oph\#11 (Jayawardhana \& Ivanov 2006; Close et al. 2006), making it unlikely that the LOri167 binary would survive to an old age.

The very existence of this type of pair is very important, because it would prove that both very low mass brown dwarfs and planetary-mass objects form from fragmentation and collapse, even if the process takes place in a hierarchical process, with successive fragmentations generating binary or multiple systems. The embryo hypothesis cannot be completely ruled out as a complementary mechanism to form very low mass brown dwarfs and planetary-mass objects, but due to the evolutionary stage of LOri167 and other similar systems, it could not explain these types of binaries at their wide separation.

\section{Conclusions}

By collecting multi-wavelength photometry in the range $0.8-4.5$ micron, we have identified a very low mass visual binary in the 5 Myr Lambda Orionis cluster, separated by 5" and equivalent to $2000 \mathrm{AU}$. We have confirmed the brown dwarf nature of the primary, whose mass is in the range 15-20 $M_{\text {jup }}$ and $T_{\text {eff }}=$ $2125 \mathrm{~K}$. The secondary, with $T_{\text {eff }}=1750 \mathrm{~K}$, would have a mass in the range $7-13 M_{\text {jup }}$ if it were a member of the cluster. This system is very important since it imposes strong constraints on the proposed formation scenarios for brown dwarfs, such as turbulent fragmentation (Padoan \& Nordlund 2004), ejection from multiple proto-stellar systems (Reipurth \& Clarke 2001; or Bate et al. 2002), or a photoevaporation of massive pre-stellar cores (Whitworth \& Zinnecker 2004).

Acknowledgements. This research has been funded by Spanish grants MEC/ESP2004-01049, MEC/Consolider-CSD2006-0070, and CAM/PRICIT-S$0505 / \mathrm{ESP} / 0361$.

\section{References}

Allard, F., Hauschildt, P. H., Alexander, D. R., Tamanai, A., \& Schweitzer, A. 2001, ApJ, 556, 357

Allard, N. F., Allard, F., Hauschildt, P. H., Keilkopf, J. F., \& Machin, L. 2003, A\&A, 411, L473 
Artigau, E., Lafrenir̀e, D., Doyon, R., et al. 2007, ApJL, in press [arXiv: astro-ph/0702647]

Baraffe, I., Chabrier, G., Allard, F., \& Hauschildt, P. H. 1998, A\&A, 337, 403

Baraffe, I., Chabrier, G., Allard, F., \& Hauschildt, P. H. 2002, A\&A, 382, 563

Barrado y Navascués, D., Stauffer, J. R., Bouvier, J., et al. 2004, ApJ, 610, 1064

Barrado y Navascués, D., Stauffer, J. R., \& Bouvier, J. 2005a, The Initial Mass Function 50 Years Later, ASSL, 327, 133

Barrado y Navascués, D., Huélamo, N., \& Morales-Calderón, M. 2005b, AN, 326,981

Barrado y Navascués, D., Stauffer, J. R., Morales-Calderón, M., et al. 2007, ApJ, in press

Basri, G., Mohanty, S., Allard, F., et al. 2000, ApJ, 538, 363

Bate, M. R., Bonnell, I. A., \& Bromm, V. 2002, MNRAS, 332, L62

Billères, M., Delfosse, X., Beuzit, J.-L., et al. 2005, A\&A, 440, L55

Bouy, H., Martin, E. L., Brandner, W., et al. 2006, A\&A, 451, 177

Caballero, J. A., Martin, E. L., Dobbie, P. D., \& Barrado y Navascués, D. 2006, A\&A, 460, 635
Caballero, J. A. 2007, A\&A, 462, L61

Chabrier, G., Baraffe, I. G., Allard, F., \& Hauschildt, P. H. 2000, ApJ, 542, 464

Chauvin, G., et al. 2004, A\&A, 425, L29

Close, L. M., Siegler, N., Freed, M., \& Biller, B. 2003, ApJ, 587, 407

Close, et al. 2006, ApJ, in press [arXiv: astro-ph/0608574]

Dolan, C. J. \& Mathieu, R. D. 1999, AJ, 118, 2409

Dolan, C. J., \& Mathieu, R. D. 2001, AJ, 121, 2124

Kraus, A. L., \& Hillenbrand, L. A. 2007, ApJ, in press [arXiv:astro-ph/0702545]

Jayawardhana, R., \& Ivanov, V. 2006, Science, 313, 1279

Kraus, A. L., White, R. J., \& Hillenbrand, L. A. 2005, ApJ, 633, 452

Kraus, A. L., White, R. J., \& Hillenbrand, L. A. 2006, ApJ, 649, 306

Luhman, K. L. 2004, ApJ, 614, 398

Luhman, K. L. 2005, ApJ, 633, L41

Padoan, P., \& Nordlund 2004, ApJ, 617, 559

Reipurth, B., \& Clarke, C. 2001, AJ, 122, 432

Whitworth, A. P., \& Zinnecker, H. 2004, A\&A, 427, 299 